\title{
The Pathogenesis of Candida Infections in a Human Skin Model: Scanning Electron Microscope Observations
}

\author{
A. Raz-Pasteur, ${ }^{1,2}$ Y. Ullmann, ${ }^{1,3}$ and I. Berdicevsky ${ }^{1}$ \\ ${ }^{1}$ Departement of Microbiology, The Ruth and Bruce Rappaport Faculty of Medicine, Technion-Institute of Technology, \\ P.O. Box 9649, 31096 Haifa, Israel \\ ${ }_{2}^{2}$ Infectious Diseases Unit, Rambam Health Care Campus, 31096 Haifa, Israel \\ ${ }^{3}$ Department of Plastic Surgery, Rambam Health Care Campus, 31096 Haifa, Israel
}

Correspondence should be addressed to I. Berdicevsky, israelab@tx.technion.ac.il

Received 16 May 2011; Accepted 13 June 2011

Academic Editor: C. R. Daniel

Copyright (๑) 2011 A. Raz-Pasteur et al. This is an open access article distributed under the Creative Commons Attribution License, which permits unrestricted use, distribution, and reproduction in any medium, provided the original work is properly cited.

Cutaneous candidiasis is an opportunistic infection that arises, in most cases, from endogenous, saprophytic candidal blastospores that selectively colonize oral, gastrointestinal, vaginal, and cutaneous epithelium. Candida albicans has been regarded as the most common causative agent in human fungal infections. However, other Candida species have become a significant cause of infection. Scanning electron microscope (SEM) observations were used to analyze the capability of C. albicans, C. tropicalis, and C. parapsilosis to adhere to human skin model, used in this study, which was found to mimic the human skin in vivo. The skin sections were inoculated with low and high concentration of the yeasts and followed for 1 and 5 days; then they were viewed by SEM. The electron microscopy observations revealed that all three yeasts tested adhered to the skin but C. albicans covered the entire skin model to a higher extent than C. tropicalis or C. parapsilosis. Mucin-like material coated the blastoconidia mainly in $C$. albicans. All Candida species have shown characteristics resembling biofilm formation. The use of human skin sections for ex vivo evaluation of adherence of various yeasts may partially explain the predominance of C. albicans in cutaneous pathogenicity.

\section{Introduction}

Yeasts are unicellular fungi that typically reproduce by budding.

Candidosis is an infection caused by the yeast Candida albicans or other Candida species.

Candida albicans is the principal fungal infectious agent in human infection.

Superficial infections of skin and mucous membranes are the most common types of candidal infections of the skin [1].

Cutaneous candidiasis is an opportunistic infection that arises, in most cases, from endogenous, saprophytic Candida blastospores that selectively colonize oral, gastrointestinal, vaginal, and cutaneous epithelium [2-5].

Under various environmental conditions, Candida blastospores may undergo mycelial transformation, invade epithelial tissue, and evoke a complement-dependent neutrophil-mediated acute inflammatory response characterized by subcorneal pustules $[6,7]$.
Initial and prerequisite events in cutaneous candidiasis should, hypothetically, include colonization of epithelial surfaces with pathogenic species of Candida.

Candida tropicalis, Candida parapsilosis, Candida krusei, Candida guilliermondii and are less common in causing human disease.

It has been well demonstrated that specific Candida species, notably $C$. albicans, selectively adhere to vaginal and buccal mucosal cells [8].

This selective adherence is thought to contribute in part to predominance of $C$. albicans colonization and infection in human hosts [9].

Most candidal species are known to produce virulence factors including protease factors.

The ability of the various yeast forms to adhere to the underlying epithelium is an important step in the production of hyphae and tissue penetration [1].

In cases in which the epithelial barrier or host immunity is impaired, Candida species may cause opportunistic infections of the skin and mucosal cavities. 
Initial events of cutaneous candidiasis include adherence of blastoconidia to epithelial cell surface, fungal proliferation and colonization, and invasion of epithelial tissue [10].

In vitro studies show that adherence to corneocytes [11] and mucosal cells [12] in most cases is associated with pathogenic species.

In rodent models, it was shown that the same species are capable of hyphal invasion of corneocytes and stratum corneum to produce infection [2].

Non pathogenic species adhere poorly to corneocytes or mucosal cells [2-7] and do not invade the stratum corneum in animal models [13].

Some studies concerning the infectivity and pathogenicity of Candida used animals models for investigating pathogenic mechanisms $[6,7]$.

As an alternative to these models, other researchers used noninvasive methods obtaining separate corneocyte cells and used them as skin surface for Candida infection $[5,6,9]$.

In order to characterize and compare initial adherence of C. albicans, C. tropicalis, and C. parapsilosis, we have used a skin model of ex vivo skin sections. We infected those skin sections with the 3 species of Candida mentioned above and examined them by scanning electron microscopy [14].

In the present study, we are reporting observations on the way Candida infect the stratum corneum in a skin model, which is a unique model that mimics the human skin.

\section{Materials and Methods}

2.1. Skin Sections. Normal thigh skin was harvested from skin surgically excised from women that underwent abdominoplasty. All specimens were prepared by putting pieces of skin, approximately $1 \mathrm{~cm}^{2}$, with full epidermal thickness into small $(60 \mathrm{~mm})$ petri dishes. The skin pieces were immersed in cold sterilized skin graft fluid (SGF) that was proved as adequate storage medium, which prolongs ex vivo skin viability for 2-3 weeks. Whenever possible, the skin was used within $1 \mathrm{~h}$ of its removal.

2.2. Skin Preservation (Storage) Medium. Skin graft fluid (SGF) at pH 6.4 was prepared using concentrated balanced salt solution $8.0 \mathrm{~mL}$ which is composed of $\mathrm{NaCl} 8.0 \mathrm{~g} / \mathrm{l}$, $\mathrm{KCl} 0.4 \mathrm{~g} / \mathrm{l}$, dibasic sodium phosphate $7 \mathrm{H}_{2} \mathrm{O} 0.0875 \mathrm{~g} / \mathrm{l}$, nonbasic potassium phosphate $0.0625 \mathrm{~g} / \mathrm{l}$, magnesium sulphate $7 \mathrm{H}_{2} \mathrm{O} 0.2 \mathrm{~g} / \mathrm{l}$, and dextrose anhydrous $1.0 \mathrm{~g} / \mathrm{l}$, all dissolved in $\mathrm{H}_{2} \mathrm{O}$ and sterilized, normal human plasma $\mathrm{AB}$ or $\mathrm{A}$ $20.0 \mathrm{~mL}$ and $0.5 \%$ neomycin sulphate in $72.0 \mathrm{~mL}$ of distilled water.

2.3. Skin Viability. The criteria for determining the viability of the skin were based on histological tests detailed by Peled et al. [15]. The extent of epidermal/dermal attachment at the interface line was observed.
2.4. Skin Inoculation. Fifty microliter of each fungal strain were spread equally over the skin surface (stratum corneum). The samples were incubated for 1 day to 5 days, at $30^{\circ} \mathrm{C}$. The storage medium (SGF) was changed daily. Two different concentrations of blastoconidia, $10^{4} \mathrm{~mL}^{-1}$ (low) and $10^{6} \mathrm{~mL}^{-1}$ (high) in triplicates were used. The inoculum was prepared from a plate containing Sabouraud medium and incubated for 48 hours. Thereafter the proper concentrations were estimated by viable counting.

Controls: skin sections without Candida were incubated under similar conditions.

2.5. Electron Microscopy. Scanning electron microscopy was used for examining the inoculated skin specimens.

Scanning Electron Microscopy (SEM). specimens were fixed overnight in $2.5 \%$ glutaraldehyde in $0.1 \mathrm{M}$ sodium cacodylate buffer $(\mathrm{pH} 7.2)$ at $4^{\circ} \mathrm{C}$, rinsed three times with PBS; and postfixed with $2 \%$ osmium tetraoxide for $2 \mathrm{~h}$. Samples were then dehydrated in graded concentrations of ethanol (25-100\%) and air-dried. The samples were viewed on an HR-SEM, ultraplus, Carl Zeiss SMT, Gemini.

2.6. Cultures. Clinical isolates of Candida albicans, Candida tropicalis, and Candida parapsilosis were obtained from Microbiology Laboratory of Rambam Health Care Campus and identified by sugar assimilation and fermentation criteria. They were maintained on Sabouraud dextrose agar slants containing $0.05 \mathrm{mg} / \mathrm{mL}$ chloramphenicol.

\section{Results}

3.1. Candida albicans. C. albicans adhered to human epidermis in greater numbers than the other species at every time point studied (after 24 hours and after 5 days) and with both concentrations $\left(10^{4} \mathrm{~mL}^{-1}\right.$ and $\left.10^{6} \mathrm{~mL}^{-1}\right)$. Large amount of C. albicans proliferated on the skin, already after 1 day at low concentration (Figure 1(a)). A higher number of yeasts appeared in higher concentration (Figure 1(b)). After 1 day and to a greater extent after 5 days, most of the $C$. albicans blastoconidia were coated with amorphous material linking adjacent blastoconidia and blastoconidia with the corneocyte surface.

The material had a strand-like appearance (in past studies "cohesion") (Figures 1(a) and 1(b)).

Pseudohyphae elements are observed after 1 day and after 5 days (Figures 1 (b) and $1(\mathrm{c})$ ).

After 5 days, huge amounts of blastoconidia are shown, covering almost the entire skin model, and the blastoconidia are embedded in a layer that resembles biofilm (Figures 1(d), $1(\mathrm{e})$, and $1(\mathrm{f}))$.

3.2. Candida tropicalis. Low amounts of blastoconidia are observed with $C$. tropicalis in comparison to $C$. albicans (Figure 2(a)).

After 5 days at the higher concentrations the blastoconidia were coated with the material linking adjacent 


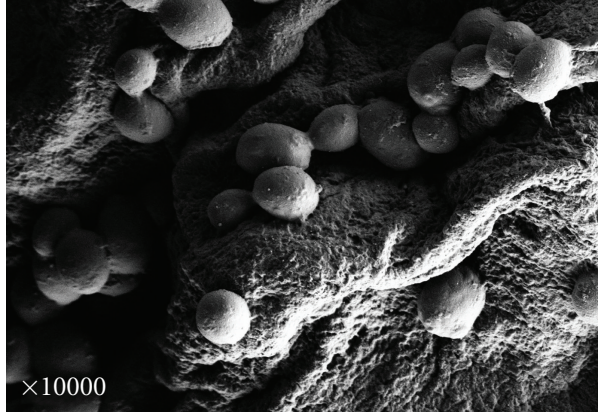

(a) 1 day $10^{4} \mathrm{~mL}^{-1}$

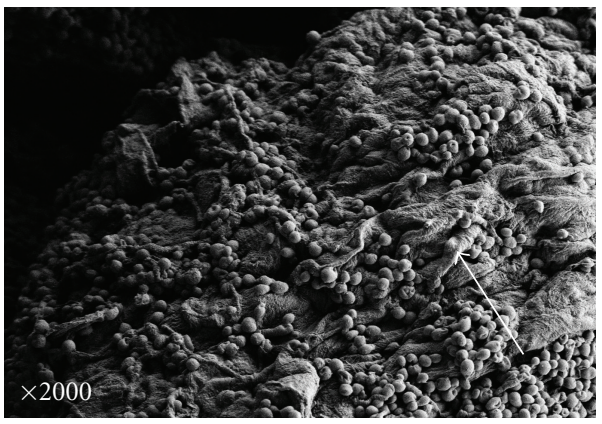

(c) 5 days $10^{4} \mathrm{~mL}^{-1}$

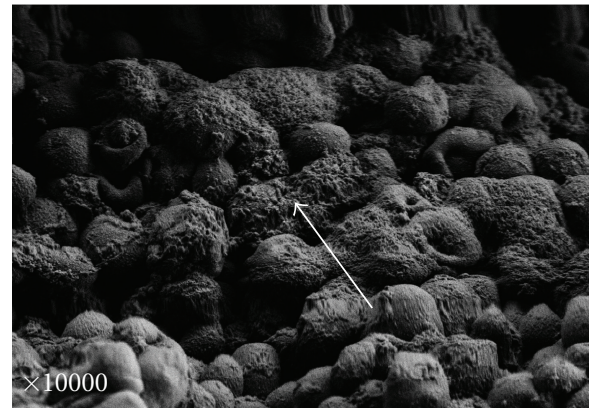

(e) 5 days $10^{6} \mathrm{~mL}^{-1}$

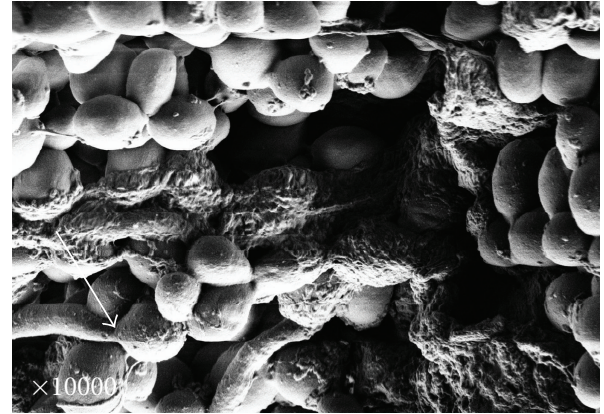

(b) 1 day $10^{6} \mathrm{~mL}^{-1}$

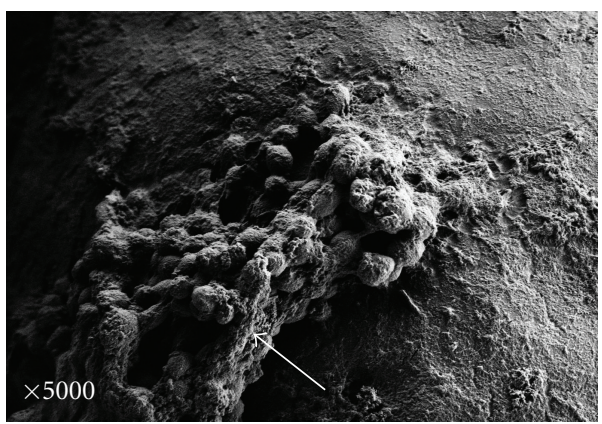

(d) 5 days $10^{6} \mathrm{~mL}^{-1}$

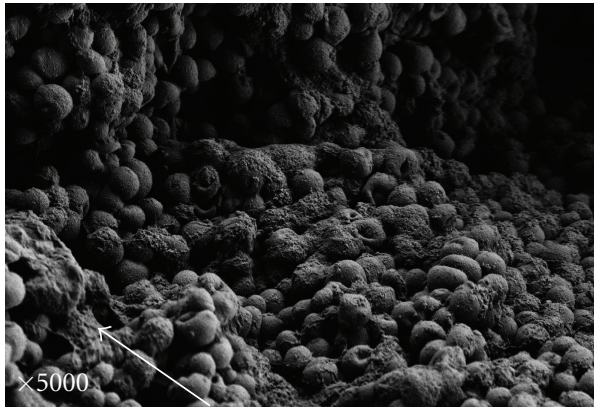

(f) 5 days $10^{6} \mathrm{~mL}^{-1}$

FIgURE 1: SEM of Candida albicans. Scanning electron micrographs (SEM) of Candida albicans after 1 day at $10^{4} \mathrm{~mL}^{-1}$ (a) and $10^{6} \mathrm{~mL}^{-1}$ concentrations (b) and after 5 days of incubation at $10^{4} \mathrm{~mL}^{-1}$ (c) and at $10^{6} \mathrm{~mL}^{-1}$ concentration of blastospors ((d), (e), and (f)). Large amounts of $C$. albicans are connected to the damaged skin. After 5 days the yeasts were covered probably by biofilm.

blastoconidia (Figures 2(e) and 2(f)), and they were covered probably with a biofilm (Figure 2(d)).

Pseudohyphae are observed after 1 day at the higher concentration and also after 5 days (Figures 2(b) and 2(c))

3.3. Candida parapsilosis. After 1 day at low and high concentrations, only few blastoconidia were observed (Figures $3(\mathrm{a})$ and $3(\mathrm{~b})$ ).

After 5 days of incubation, more blastoconidia adhered in both concentrations, some kind of "threads" are shown, and the cells are embedded in the surface layer (Figures 3(c) and $3(\mathrm{~d})$ ).

After 1 day we were able to see characteristics that resemble minimal biofilm formation in all Candida species with no difference in both concentrations; this biofilm layer increased significantly after 5 days in all the species (Figures 1(c), 1(d), 2(d), 2(e), 3(c), and 3(d)).

3.4. Control Skin. After 1 day and after 5 days we observed normal epidermis (Figures 4(a) and 4(b)).

\section{Discussion}

In this study on the human skin ex vivo model, we demonstrated that $C$. albicans exhibited marked adherence to the skin in comparison to C. parapsilosis and C. tropicalis at two time points and in different concentrations.

Adhesion is provided partially by mucopolysaccharide molecules and can be evaluated qualitatively by microscopic examination of interactions between the yeast 


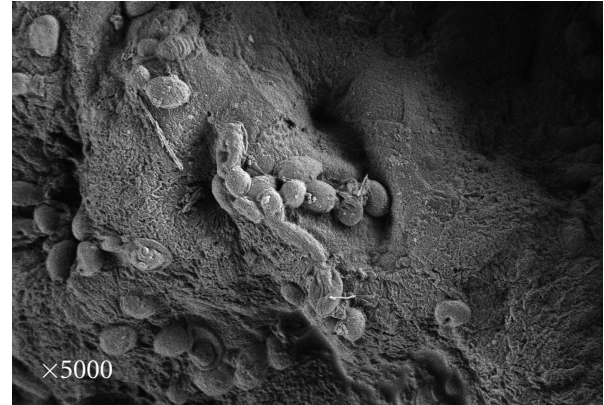

(a) 1 day $10^{4} \mathrm{~mL}^{-1}$

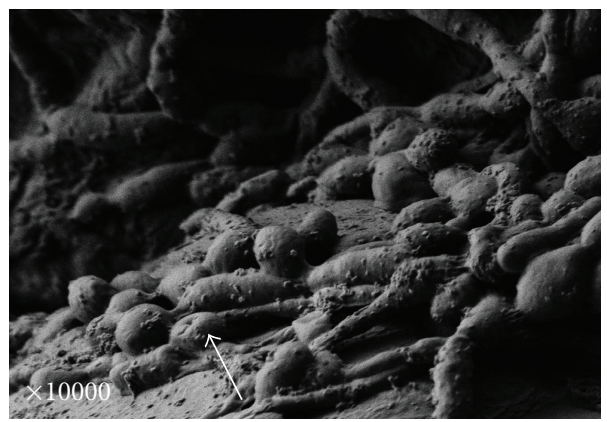

(c) 5 days $10^{4} \mathrm{~mL}^{-1}$

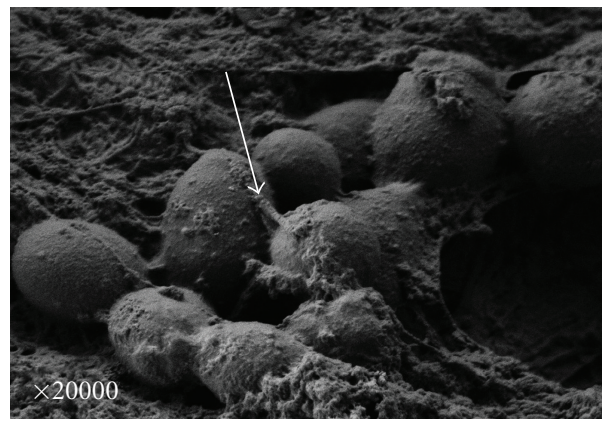

(e) 5 days $10^{6} \mathrm{~mL}^{-1}$

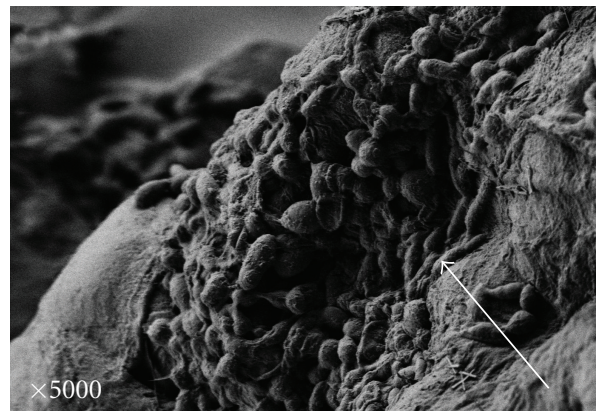

(b) 1 day $10^{6} \mathrm{~mL}^{-1}$

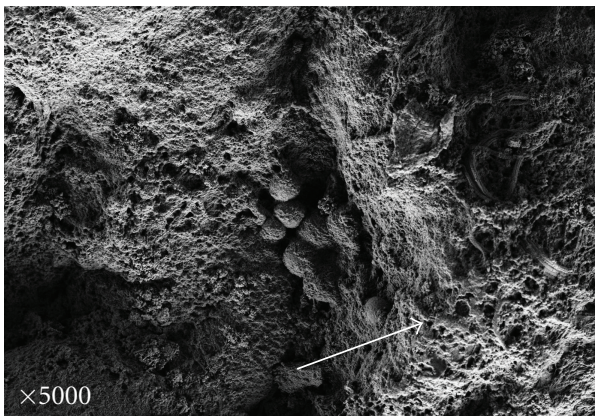

(d) 5 days $10^{6} \mathrm{~mL}^{-1}$

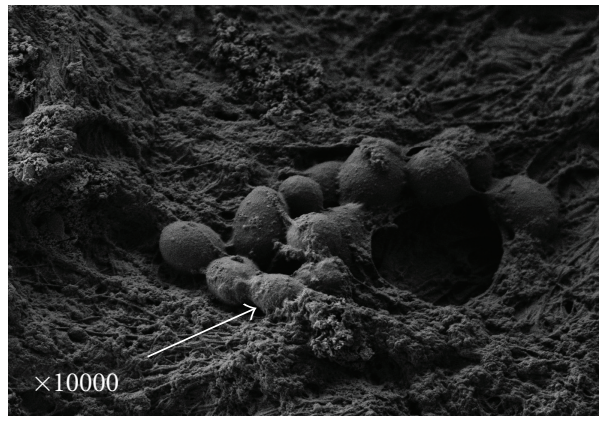

(f) 5 days $10^{6} \mathrm{~mL}^{-1}$

Figure 2: SEM of Candida tropicalis. SEM of Candida tropicalis after 1 day at $10^{4} \mathrm{~mL}^{-1}$ (a) and $10^{6} \mathrm{~mL}^{-1}$ (b). After 5 days at $10^{4} \mathrm{~mL}^{-1}$ (c) and $10^{6} \mathrm{~mL}^{-1}((\mathrm{~d}),(\mathrm{e})$, and $(\mathrm{f}))$ the skin is damaged and cells are adhered and "integrated" into the skin.

and the epithelium or quantitatively by measuring the specific adhesion of Candida to cultured epithelial cells $[16,17]$.

C. albicans selectively adheres to buccal and vaginal epithelial cells in humans, and adherence may play a critical role in the pathogenesis of mucocutaneous candidiasis [18].

In experimental rodent cutaneous candidiasis using $C$. albicans, C. stellatoidea, C. tropicalis, C. parapsilosis, and C. $k$ rusei, the only pathogenic species after topical application to intact epidermis were C. albicans and C. stellatoidea [16]; C. tropicalis, C. parapsilosis, and C. krusei exhibited minor or negligible adherence to epidermal corneocytes and were nonpathogenic to skin. Those Candida species are occasionally systemic pathogens associated with septicemia $[16,18]$.
In concordance with past studies, the present study ultrastructural visualization of adherence demonstrated amorphous material coating blastoconidia, mainly of Candida albicans and minimally of C. parapsilosis and C. tropicalis.

In those past studies this material was called cohesin. This cohesin was found as a film of loosely adherent strandlike material on the corneocyte surface of newborn mouse skin, in association with adherent blastoconidia, and was seen only with C. albicans and C. stellatoidea and not with $C$. parapsilosis and C. tropicalis [10].

In our study, all Candida species have shown characteristics resembling biofilm formation. There is evidence that the majority of Candida species (albicans and non-albicans), have the capacity of producing significant amounts of biofilm 


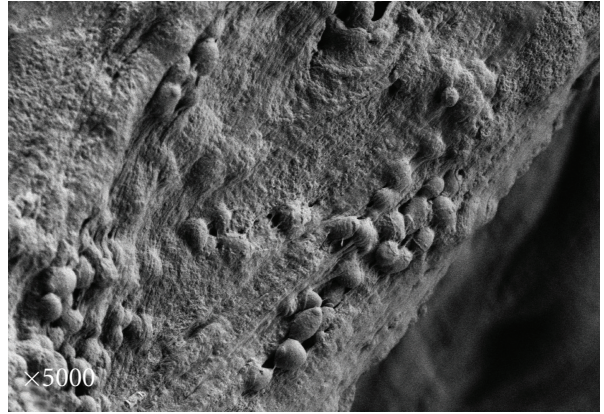

(a) 1 day $10^{4} \mathrm{~mL}^{-1}$

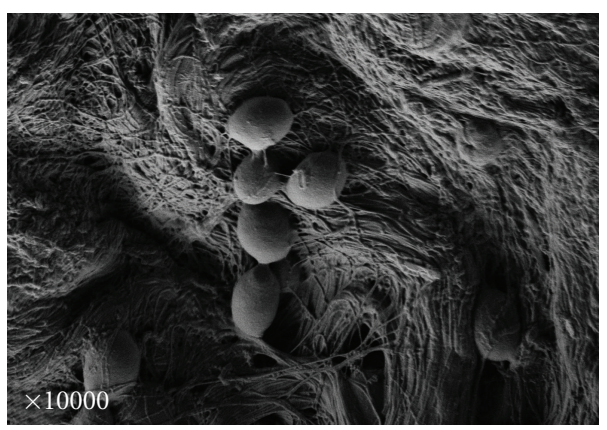

(c) 5 days $10^{4} \mathrm{~mL}^{-1}$

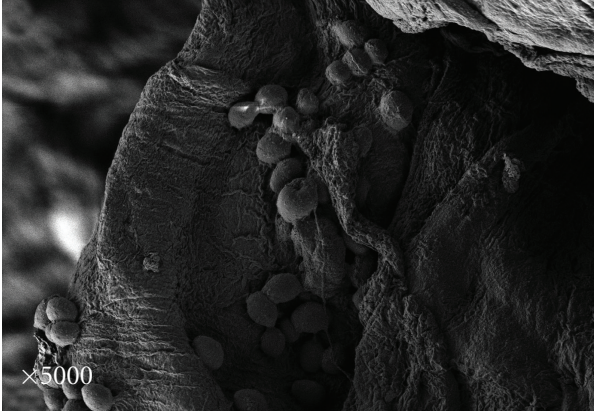

(b) 1 day $10^{6} \mathrm{~mL}^{-1}$

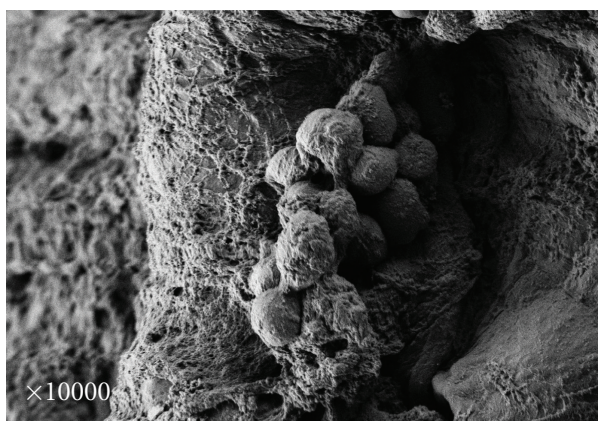

(d) 5 days $10^{6} \mathrm{~mL}^{-1}$

FIgure 3: SEM of Candida parapsilosis. SEM of Candida parapsilosis after 1 day incubation at $10^{4} \mathrm{~mL}^{-1}$ (a) and $10^{6} \mathrm{~mL}^{-1}$ (b) and after 5 days at $10^{4} \mathrm{~mL}^{-1}(\mathrm{c})$ and at $10^{6} \mathrm{~mL}^{-1}(\mathrm{~d})$. Although the adhesion is well defined, only small amounts of yeasts are observed. The skin is damaged and the cells are embedded by thread-like appendices.

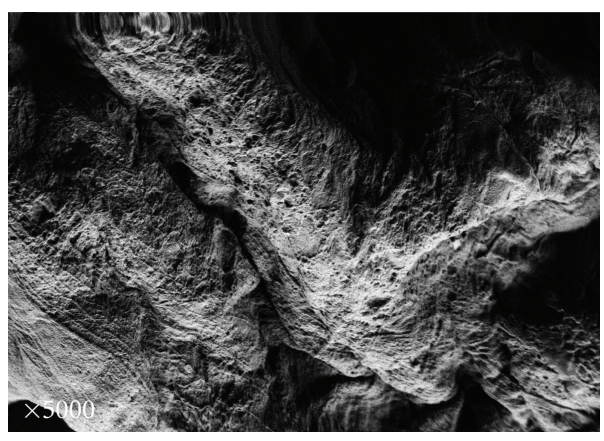

(a) 1 day

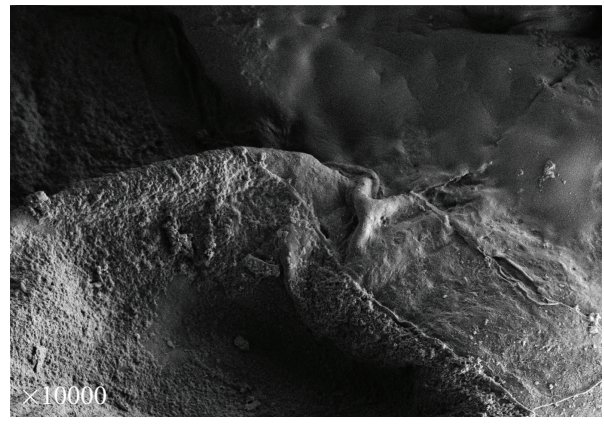

(b) 5 days

FIGURE 4: SEM of control skin. SEM of normal skin after 1 day and 5 days ((a), (b)). The skin without treatment served as control. The skin is well preserved.

and this capacity may reflect the pathogenic potential of the isolates [19].

\section{References}

[1] N. S. Scheinfeld and M. C. Lambiase, "Candidiasis, Cutaneous," http://emedicine.medscape.com/ article/ 1090632followup. updated oct 2010.

[2] T. M. Arendorf and D. M. Walker, "The prevalence and intraoral distribution of Candida albicans in man," Archives of Oral Biology, vol. 25, no. 1, pp. 1-10, 1980.
[3] A. G. E. Barlow and F. W. Chattaway, "Observations on the carriage of Candida albicans in man," British Journal of Dermatology, vol. 81, no. 2, pp. 103-106, 1969.

[4] Y. M. Clayton and W. C. Noble, "Observations on the epidemiology of Candida albicans," Journal of Clinical Pathology, vol. 19, no. 1, pp. 76-78, 1966.

[5] F. C. Odds, Candida and Candidosis, University Park Press, Baltimore, Md, USA, 1979.

[6] T. L. Ray and K. D. Wuepper, "Experimental cutaneous candidiasis in rodents," Journal of Investigative Dermatology, vol. 66, no. 1, pp. 29-33, 1976. 
[7] T. L. Ray and K. D. Wuepper, "Experimental cutaneous candidiasis in rodents. II. Role of the stratum corneum barrier and serum complement as a mediator of a protective inflammatory response," Archives of Dermatology, vol. 114, no. 4, pp. 539-543, 1978.

[8] L. P. Samaranayake and T. W. MacFarlane, "The adhesion of the yeast Candida albicans to epithelial cells of human origin in vitro," Archives of Oral Biology, vol. 26, no. 10, pp. 815-820, 1981.

[9] J. C. Lee and R. D. King, "Characterization of Candida albicans adherence to human vaginal epithelial cells in vitro," Infection and Immunity, vol. 41, no. 3, pp. 1024-1030, 1983.

[10] T. L. Ray and C. D. Payne, "Scanning electron microscopy of epidermal adherence and cavitation in murine candidiasis: a role for Candida acid proteinase," Infection and Immunity, vol. 56, no. 8, pp. 1942-1949, 1988.

[11] H. Remold, H. Fasold, and H. Staib, "Purification and characterization of a proteolytic enzyme from Candida albicans," Biochimica et Biophysica Acta, vol. 167, no. 2, pp. 399-406, 1968.

[12] R. D. King, J. C. Lee, and A. L. Morris, "Adherence of Candida albicans and other Candida species to mucosal epithelial cells," Infection and Immunity, vol. 27, no. 2, pp. 667-674, 1980.

[13] T. L. Ray and K. D. Wuepper, "Experimental cutaneous candidiasis in rodents," Journal of Investigative Dermatology, vol. 66, no. 1, pp. 29-33, 1976.

[14] L. Duek, G. Kaufman, Y. Ulman, and I. Berdicevsky, "The pathogenesis of dermatophyte infections in human skin sections," Journal of Infection, vol. 48, no. 2, pp. 175-180, 2004.

[15] I. J. Peled, E. Notea, and E. Lindenbaum, "Prolonged skin graft preservation with keratinocyte culture medium," European Journal of Plastic Surgery, vol. 14, no. 5, pp. 232-234, 1991.

[16] C. M. Bendel, "Colonization and epithelial adhesion in the pathogenesis of neonatal candidiasis," Seminars in Perinatology, vol. 27, no. 5, pp. 351-424, 2003.

[17] J. A. M. S. Jayatilake, "A review of the Ultrastructural Features of Superficial Candidiasis," Mycopathologia, vol. 171, pp. 235250, 2010.

[18] T. L. Ray, K. B. Digre, and C. D. Payne, "Adherence of Candida species to human epidermal corneocytes and buccal mucosal cells: correlation with cutaneous pathogenicity," Journal of Investigative Dermatology, vol. 83, no. 1, pp. 37-41, 1984.

[19] V. Mohandas and M. Ballal, "Distribution of Candida Species in different clinical samples and their virulence: biofilm formation, proteinase and phospholipase production," Journal of Global Infectious Diseases, vol. 3, no. 1, pp. 4-8, 2011. 


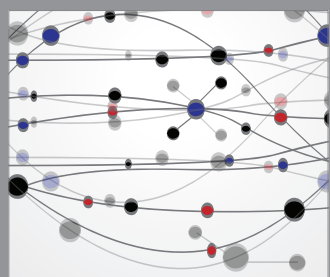

The Scientific World Journal
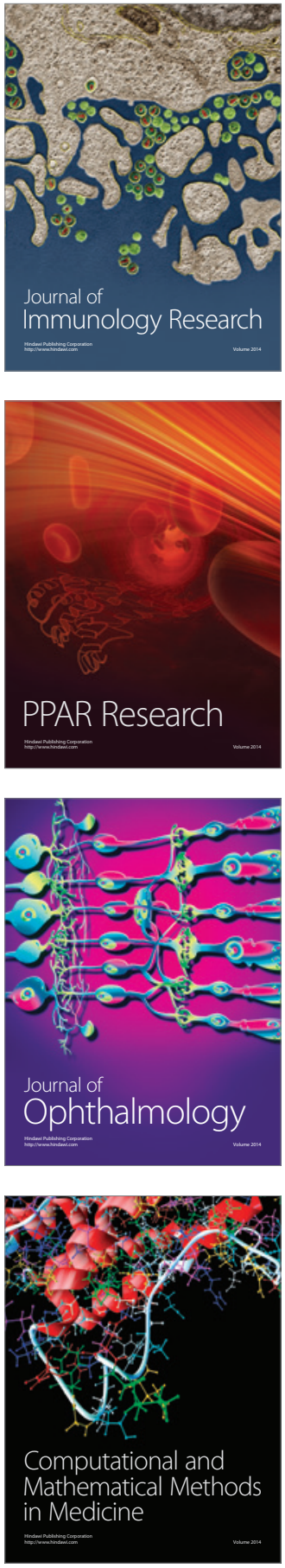

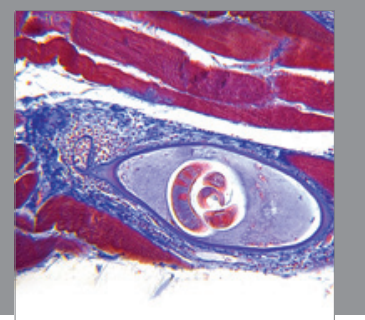

Gastroenterology

Research and Practice
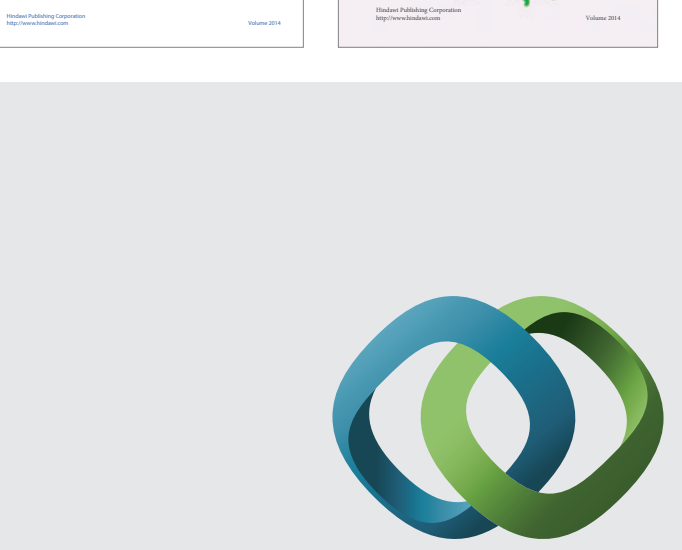

\section{Hindawi}

Submit your manuscripts at

http://www.hindawi.com
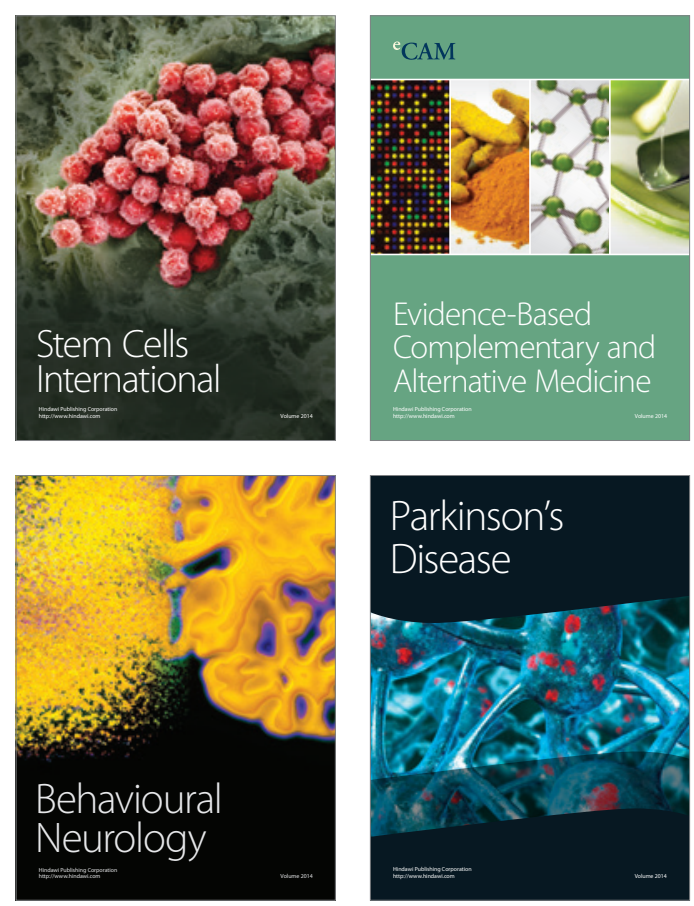

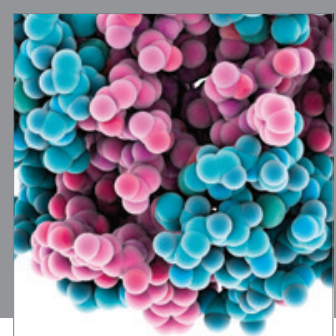

Journal of
Diabetes Research

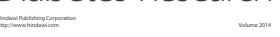

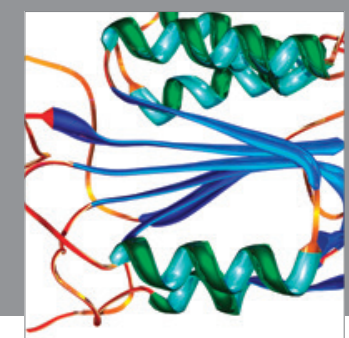

Disease Markers
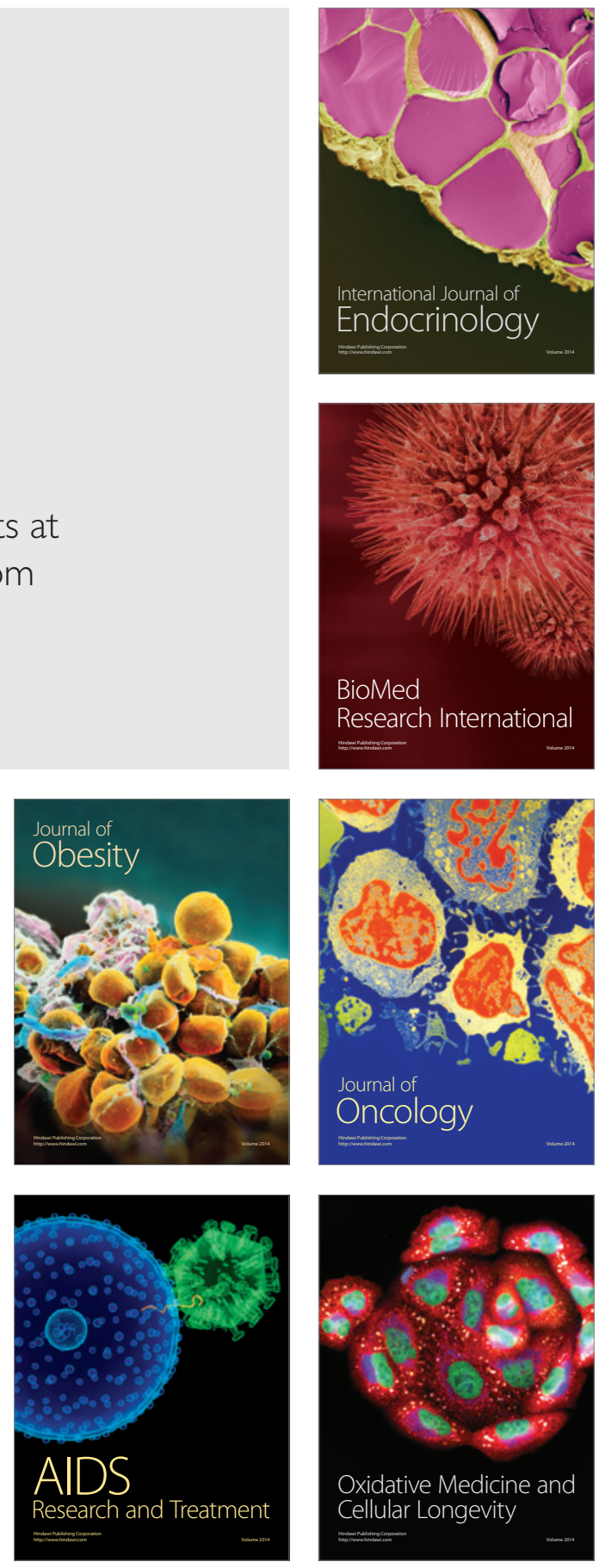